\title{
Las representaciones sociales
}

\section{sobre el concepto de género en personas} que ejercen cargos de gestión en la Facultad de Filosofía Humanidades y Arte y la Facultad de Ingeniería
de la Universidad Nacional de San Juan

DOI: 10.36888/udual.universidades.2018.77.174

\section{Resumen}

El presente artículo subsume algunos hallazgos del Proyecto de Investigación "Diseño y atención a las oportunidades de género y educación superior” en la Universidad Nacional de San Juan.

El propósito de este artículo es problematizar las Representaciones Sociales (RS) acerca del concepto de género de las personas que ejercen la gestión académica en el período 2016-2020 en la Facultad de Ingeniería y la Facultad de Filosofía Humanidades y Artes pertenecientes a la UNSJ. Este estudio se realiza desde un enfoque de género y con un abordaje metodológico que articula elementos cuantitativos y cualitativos lo que nos permitió poner en valor y reforzar el proceso de validación/discusión de los datos a través del lenguaje de los/las entrevistados/as y la reconstrucción permanente del trabajo de investigación.

Palabras Clave: Género, Educación superior, Representaciones Sociales.

POR VALERIA GILI DIEZ / FRANCO ALEXIS BARBOZA PIRAN / MARIANA GUERRA PÉREZ Licenciada y Profesora en Sociología. Doctoranda en la Universidad Nacional de Córdoba con el tema de investigación "Los procesos de transición a la vida adulta: itinerarios familiares, educativos y laborales de jóvenes chacareros sanjuaninos, Argentina". Docente, investigadora y extensionista de la FACSO-FCEFyN de la Universidad Nacional de San Juan. Miembro del Grupo de Articulación en Género y Educación Superior (GAGES)-UNSJ. Líneas de investigación: Género, Educación Superior, Salud. valeriagili@hotmail.com / Estudiante de 5to año de la carrera de Sociología. Miembro del Grupo de Articulación en Género y Educación Superior (GAGES)-UNSJ. Líneas de investigación: Género, Educación Superior, Salud. francoabarboza@gmail.com / Licenciada en Filosofía. Doctoranda en Filosofía por la UNC. Docente en cátedra Filosofía y Conocimiento y Problemática Filosófica en la FFHA-UNSJ. Becaria doctoral de CONICET-UNSJ, con el tema de investigación "Una alternativa epistemológica-semióticapolítica situada desde los feminismos decoloniales, en diálogos y tensiones con los feminismos latinoamericanos". Miembro del Grupo de Articulación en Género y Educación Superior (GAGES)-UNSJ. Líneas de investigación: Género, Educación Superior, Salud.mariananoelguerra@gmail.com 


\section{Abstract}

This article subsumes some findings of the Research Project "Design and attention to gender opportunities and higher education" at the National University of San Juan.

The purpose of this article is to problematize the Social Representations (SR) about the concept of gender of the people who exercise academic management in the period 2016-2020 in the Faculty of Engineering and the Faculty of Philosophy Humanities and Arts belonging to the UNSJ. This study is carried out from a gender perspective and with a methodological approach that articulates quantitative and qualitative elements, which allowed us to value and reinforce the process of validation / discussion of the data through the language of the interviewees and the permanent reconstruction of the research work.

Keywords: Gender, Higher Education, Social representations.

\section{Resumo}

O presente artigo subsome alguns achados do Projeto de Pesquisa "Desenho e atenção às oportunidades de género e educação superior" na Universidad Nacional de San Juan.

O proposito deste artigo é problematizar as Representações Sociais (RS) sobre o conceito de gênero das pessoas que exercem a gestão académica no período 2016-2020 na Facultad de Ingeniería y la Facultad de Filosofía Humanidades y Artes pertencentes à UNSJ. Neste estudo realiza-se desde um enfoque de gênero e com uma abordagem metodológica que articula elementos quantitativos e qualitativos, o que permitiu dar valor e reforçar o processo de validação/ discussão dos dados a través da linguagem dos/das entrevistados/as e a reconstrução permanente do trabalho de pesquisa.

Palabras Clave: Gênero, Educação Superior, Representações Sociais.
Este proyecto forma parte de la Red de Investigación en Diferenciales de Género en la Educación Superior Iberoamericana (2016-2017), coordinada por la Universidad de Alicante.

\section{Introducción}

El propósito de este artículo es problematizar las Representaciones Sociales (RS) acerca del concepto de género de las personas que ejercen la gestión académica en el período 2016-2020 en la Facultad de Ingeniería (FI) y la Facultad de Filosofía, Humanidades y Artes (FFHA) pertenecientes a la Universidad Nacional de San Juan (UNSJ) ${ }^{1}$. Los interrogantes que guiaron el proceso de investigación son ¿̇cuáles son las RS sobre género que poseen las personas que ejercen cargos de gestión en la FI y FFHA de la UNSJ? ¿Cuáles son las diferencias y similitudes en las RS de las personas que ocupan cargos de gestión según formación disciplinar?

Comprendemos que dichos interrogantes nos sitúan en un intersticio entre la psicología y la sociología; ya clásicos como Durkheim (2010) se referían a las representaciones colectivas. En este sentido, las RS “...conciernen a la manera cómo nosotros, sujetos sociales, aprendemos los acontecimientos de la vida diaria (...). En pocas palabras el conocimiento "espontáneo", "ingenuo", que tanto interesa en la actualidad a las ciencias sociales, ese que habitualmente se denomina conocimiento del sentido común (...). Este conocimiento se constituye a partir de nuestras experiencias, pero también de las informaciones, conocimientos, y modelos de pensamiento que percibimos y transmitimos a través de la tradición, la educación y la comunicación social (...) es un conocimiento socialmente elaborado y compartido" (Jodelet, 1988)

Este estudio se realiza desde un enfoque de género, que funciona como un prisma para develar la presencia de estructuras de poder asimétricas que asignan valores, posiciones, hábitos, diferenciales a cada uno de los géneros (Pautassi, 2011).

Históricamente, la diferenciación entre los sexos ha otorgado a hombres y mujeres una distribución de roles, atributos y funciones ligados a lo socialmente esperado para cada uno. La visión hegemónica patriarcal asigna a la mujer las funciones reproductivas, 
de cuidados, de la vida doméstica o del ámbito de lo privado y a los varones la función productiva, proveedora y ligada a los ámbitos públicos.

El concepto de género nace de las corrientes feministas y tiene como un primer objetivo: la diferenciación respecto del concepto de sexo. Este último refiere a la dimensión biológica (relacionada con lo físico genital) mientras que el género da cuenta de los atributos, funciones, roles, responsabilidades e identidades que se construyen socialmente. De ahí que, según Lamas, la categoría de género "se refiere a la construcción social y cultural que organiza nociones sobre lo 'propio' de lo masculino y de lo femenino a partir de la diferencia sexual" (Lamas, 1996 en Faur, 2004:75) ${ }^{2}$.

A lo largo de la historia, los roles, funciones y atributos ligados a las mujeres se encontraron en una relación de subordinación respecto a los varones, lo cual pone en evidencia una situación de desigualdad para ellas en diferentes ámbitos. Si el género refiere a todos aquellos atributos y roles sociales cambiantes que una sociedad le asigna a lo femenino y a lo masculino, en nuestras sociedades, este concepto giró en torno a una "construcción social desigual basada en la existencia de jerarquías entre ambos sexos, y a las consiguientes relaciones asimétricas de poder que se generan a partir de allí", (Pautassi, 2000: 89).

En Argentina, las normativas y las instituciones públicas han reconocido poco a poco la participación de las mujeres y otras minorías como sujetos de derechos. Da cuenta de ello la sanción de una serie de leyes que van en consonancia con este reconocimiento, a saber: La ley de Protección Integral a las Mujeres (26.485); la ley de Identidad de Género (26.743), la ley sobre Actos Discriminatorios (23.592), entre otras.

Sin embargo, aún persisten brechas respecto de la igualdad de oportunidades que ubican a estos grupos en una situación de desventaja a la hora de ocupar cargos públicos. Si bien los estudios muestran que la situación de las mujeres en los puestos de gestión y sus oportunidades de acceso, han avanzado de manera sostenida desde los años 60 , también se estima que las condiciones de igualdad están todavía distantes.

Las instituciones de educación superior no han sido ajenas a esta tendencia. Si bien es posible reconocer que los puestos de gestión han experimentado una sensible apertura a que sean ocupados por mujeres, eso no retrae los imaginarios culturales que sobre estos recaen. El ejercicio del liderazgo, como condición del patriarcado hegemónico, es probable que revista vigencia en instituciones universitarias insertas en sociedades que aún se encuentran atravesadas por esas características en la conformación de sus tramas sociales. Es por esto, que la relevancia del presente estudio radica en desentrañar estructuras y sentidos de aquellas personas encargadas de tomar decisiones en materia educativa y que, a nuestro entender, influyen directamente en los imaginarios sobre género en la educación superior.

La elección de nuestras unidades de análisis se fundamenta en lo anteriormente mencionado y en que la UNSJ está dando pasos significativos ${ }^{3}$ en el reconocimiento de los derechos de las mujeres y otras identidades disidentes. En este sentido, con el presente artículo buscamos conocer si las RS sobre género de las personas que ocupan cargos de gestión tienen relación con estos cambios que atraviesa la institución.

\section{Abordaje Metodológico}

En la investigación de la que se desprenden las reflexiones aquí presentes trabajamos metodológicamente con una estrategia cuantitativa, que permitió el abordaje de un número considerable de casos, en su contexto específico y atendiendo a su complejidad y diversidad. La relevancia de los datos obtenidos radica en la conformación de una base estadística sobre el personal en cargos de gestión de la UNSJ respecto a relaciones de género y gestión académica.

El trabajo cuantitativo fue simultáneamente enriquecido con el abordaje cualitativo de algunas categorías nodales de investigación, lo que permitió poner en valor y reforzar el proceso de validación/discusión de los datos a través del lenguaje de los/las entrevistados/as y la reconstrucción permanente del trabajo de investigación. "La investigación cualitativa depende fundamentalmente de la observación de los actores en su propio terreno y de la interacción con ellos en su lenguaje y con sus mismos términos implicando un compromiso con el trabajo de campo", (Vasilachis de Gialdino, 1992).

En este sentido, en la discusión de los datos se refleja una triangulación metodológica tendiente a 
superar la falsa dicotomía entre la lógica disyuntiva y la lógica dialéctica. Estas reflexiones representan un recorte de este trabajo de triangulación metodológica, con énfasis en una de sus dimensiones cualitativas: las RS. El abordaje cuantitativo de los datos permitió contextualizar y comparar las RS sobre el concepto género en dos facultades de la UNSJ.

Como instrumento de recolección de datos aplicamos encuestas, un cuestionario semi estandarizado compuesto por 16 preguntas que contienen:

- Variables base: género, estado civil y conformación familiar, dependencia institucional, cargo que desempeña.

- Preguntas cerradas: toma de decisiones, obstáculos en el desempeño del cargo, fuentes de aprendizaje en la gestión e influencia del género en el estilo de liderazgo.

- Preguntas abiertas: percepción de desigualdades de género en el acceso a cargos jerárquicos de gestión académica, percepción sobre el tiempo de acceso a dichos cargos según género, percepción sobre el estilo de liderazgo que ejerce y representación sobre el concepto de género.

En cuanto a la unidad de análisis ${ }^{4} \mathrm{y}$ a la población del estudio, cabe destacar que si bien trabajamos con el universo posible (78 casos), aquí nos circunscribimos a las personas que ocupan cargos de gestión académica durante el período 2016-2020 en la FI y la FFHA de la UNSJ (44 casos).

Este recorte se sustentó en el supuesto de investigación que sostiene que las RS sobre el concepto de género de las personas que ocupan cargos de gestión varía en función de la formación disciplinar obtenida.

En cuanto al estudio de las RS adherimos a un enfoque procesual que descansa en postulados cualitativos y que privilegia el análisis de lo social, de la cultura y de las interacciones sociales, en general. Este enfoque accede al conocimiento de las RS a través de un abordaje hermenéutico, en el que los actores son visualizados como productores de sentido. Asimismo, focaliza en la comprensión de las producciones simbólicas, de los significados y el lenguaje a través de los cuales los seres humanos construyen el mundo en que viven.

En este sentido, se abordaron las RS como una imagen que "(...) condensa un conjunto de signifi- caciones abstractas por lo cual le permite al sentido común interpretar, clasificar, y teorizar respecto de los acontecimientos de la vida cotidiana. En sentido amplio, es una forma de pensamiento social, que se traduce en un pensamiento práctico y orientador de los agentes sociales, para la comprensión, comunicación y dominio en la dimensión social, material e ideal", (Jodelet, 1988).

Las RS constituyen principios generadores de tomas de posición, ligados a inserciones sociales específicas, organizando los procesos simbólicos que intervienen en las relaciones sociales (Doise, 1986). Al operar como marco de interpretación del entorno, regulan las vinculaciones con el mundo y los otros, y orientan y organizan las conductas y las comunicaciones. Por ello tienen un papel importante en procesos tales como la difusión y asimilación de los nuevos conocimientos, la definición de las identidades personales y sociales, la expresión de los grupos y las transformaciones sociales (Pérez Rubio, 2004).

\section{Caracterización de la UNSJ, FI y FFHA}

La UNSJ cuenta con 5 facultades (Ingeniería; Filosofía, Humanidades y Artes; Ciencias Sociales; Ciencias Exactas, Físicas y Naturales; y Arquitectura, Urbanismo y Diseño) a las que asisten un total de 21,374 estudiantes, según informe de memoria anual de la UNSJ (2016-2017).

En el caso de la Facultad Filosofía, Humanidades y Artes y de Ingeniería, concentran el $43.23 \%$ de todos los estudiantes de la UNSJ (24.55\% y $18.68 \%$ respectivamente), siendo las $2^{\circ}$ y $3^{\circ}$ facultades con más estudiantes de la universidad. Asimismo, son las unidades académicas que cuentan con mayor cantidad de departamentos (10 en la FFHA y 9 en la FI) y mayor cantidad de carreras de grado (27 en la FFHA y 13 en la FI).

Del mismo modo, y probablemente debido a su amplia oferta académica, ambas facultades concentran el 56\% de los cargos docentes, el 60\% de las horas docentes, y el $67.64 \%$ de las horas docentes en cargos titulares exclusivos.

Si se considera a las personas en cargos de gestión electivos (Decano/Decana, Vicedecano/Vicedecana, 
Gráficos 1 y 2. Proporción de varones y mujeres en cargos de gestión en FFHA y FI de la UNSJ.
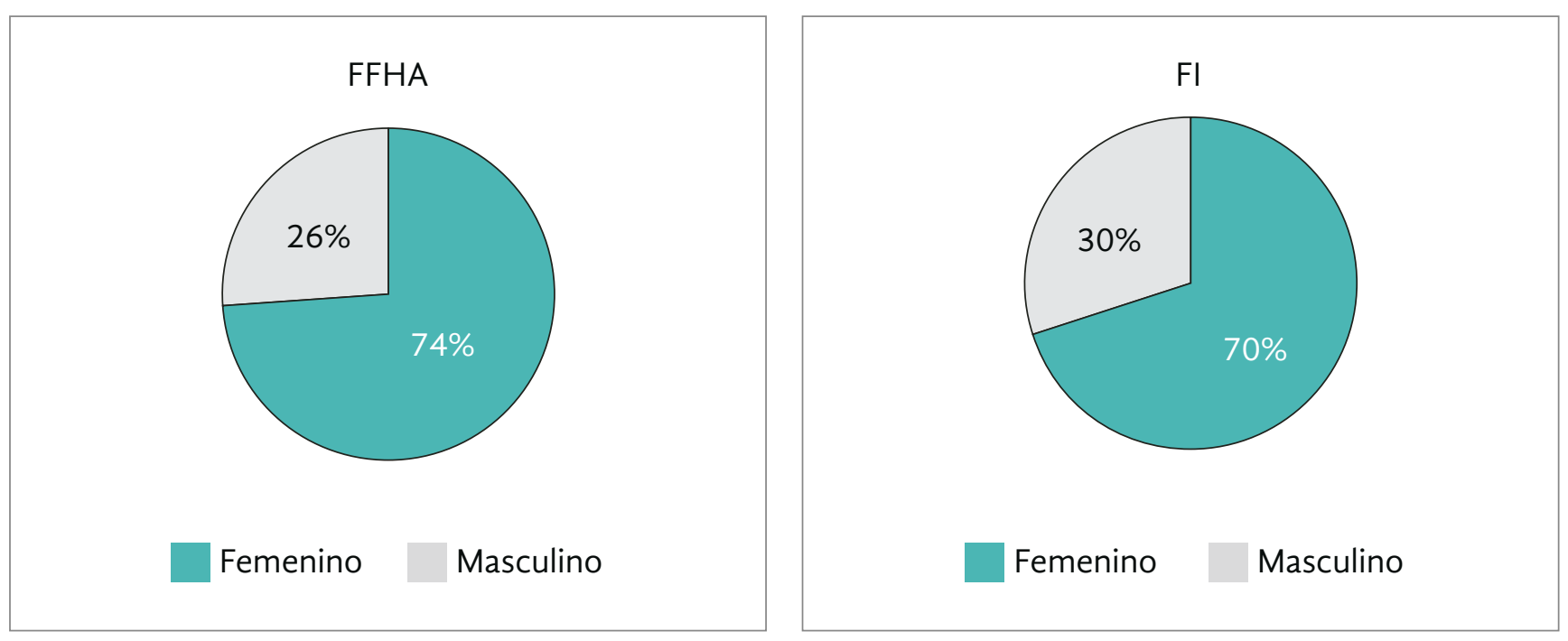

Fuente: Elaboración propia en base a la encuesta realizada a académicas/os en gestión en la UNSJ.

Gráficos 3 y 4. Cantidad de personas en gestión según cargo que ocupa, discriminado por género (FFHA y FI).
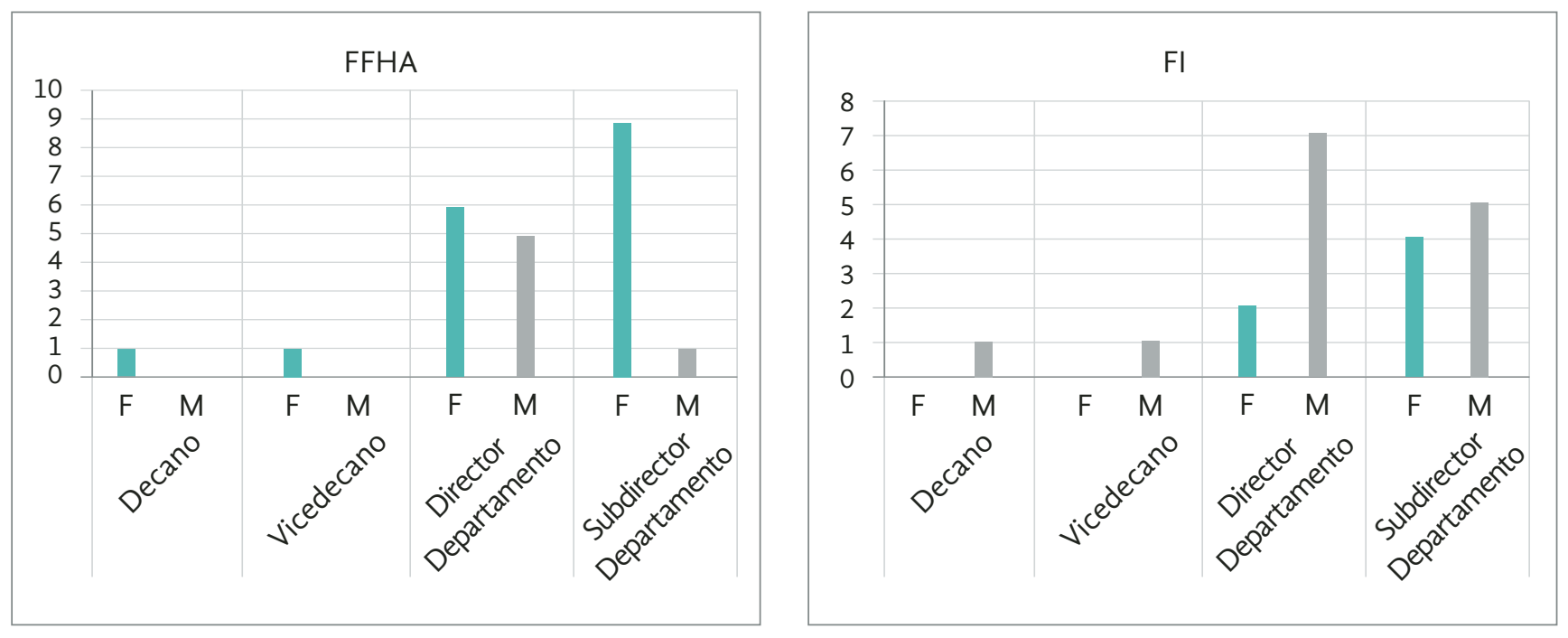

Fuente: Elaboración propia en base a la encuesta realizada a académicas/os en gestión en la UNSJ.

Director/Directora de departamento, Vicedirector/ Vicedirectora de departamento), la FI cuenta con 24 personas en estos cargos y la FFHA con 20.

En los gráficos 1 y 2 puede observarse que el $74 \%$ de los cargos de gestión electivos de la FFHA están ocupados por mujeres, mientras que sólo el $26 \%$ es ocupado por varones. De manera inversa el $70 \%$ de las personas en gestión de la FI son varones, y el 30\% mujeres.

En el precedente gráfico, podemos observar que los cargos electivos de mayor jerarquía (Decano/ Decana, Vicedecano/Vicedecana) están constituidos exclusivamente por varones en la FI y exclusivamente 
por mujeres en la FFHA. En el caso de la dirección de los departamentos, en la FFHA, estos cargos se encuentran equitativamente ocupados por varones y mujeres, mientras que en la FI la mayoría de los directores son varones y sólo 2 direcciones están lideradas por mujeres. En la FI sólo se encuentra paridad entre varones y mujeres en el cargo de subdirección de departamento, mientras que en la FFHA las subdirecciones se encuentran mayoritariamente ocupadas por mujeres, sólo una de ellas es ocupada por un varón.

En los gráficos 5 y 6 es posible apreciar la formación (o no) de posgrado de las personas que actualmente se desempeñan en cargos de gestión en la FI y la FFHA. En ambas facultades, la mayoría de las personas en gestión que no tienen título de posgrado son varones. De los/las 44 entrevistados/entrevistadas, 17 mujeres tienen formación de maestría o doctorado, principalmente en la FFHA, mientras que sólo 7 varones que cumplen funciones de gestión tienen un título de posgrado de maestría o superior.

\section{Discusión: Análisis}

de las representaciones sociales (RS) en torno al concepto de género en cargos de gestión en la UNSJ

Como se explicitó en el apartado metodológico, el trabajo cuantitativo fue simultáneamente enriquecido con el abordaje cualitativo de algunas categorías nodales de investigación. En este sentido, tomamos como insumo una de las preguntas abiertas presentes en el cuestionario de encuesta.

El interrogante apuntaba a reconstruir las significaciones y sentidos que las personas que ejercen la gestión académica en el período 2016-2020 en la FI y la FFHA pertenecientes a la UNSJ, tenían sobre el concepto de género.

Para poder comprender el universo de sentidos asociados al concepto de género es que seleccionamos como andamiaje teórico la teoría de las representaciones sociales (RS). En función de ello, presentamos aquí un esquema de categorías que apunta a comprender la heterogeneidad de perspectivas que confluyen a la hora de significar el concepto de género.

Es importante señalar que el esquema de categorías representa una herramienta analítica, un modelo
Gráficos 5 y 6. Formación de posgrado de las personas en gestión, discriminado según género

(FI y FFHA- UNSJ).
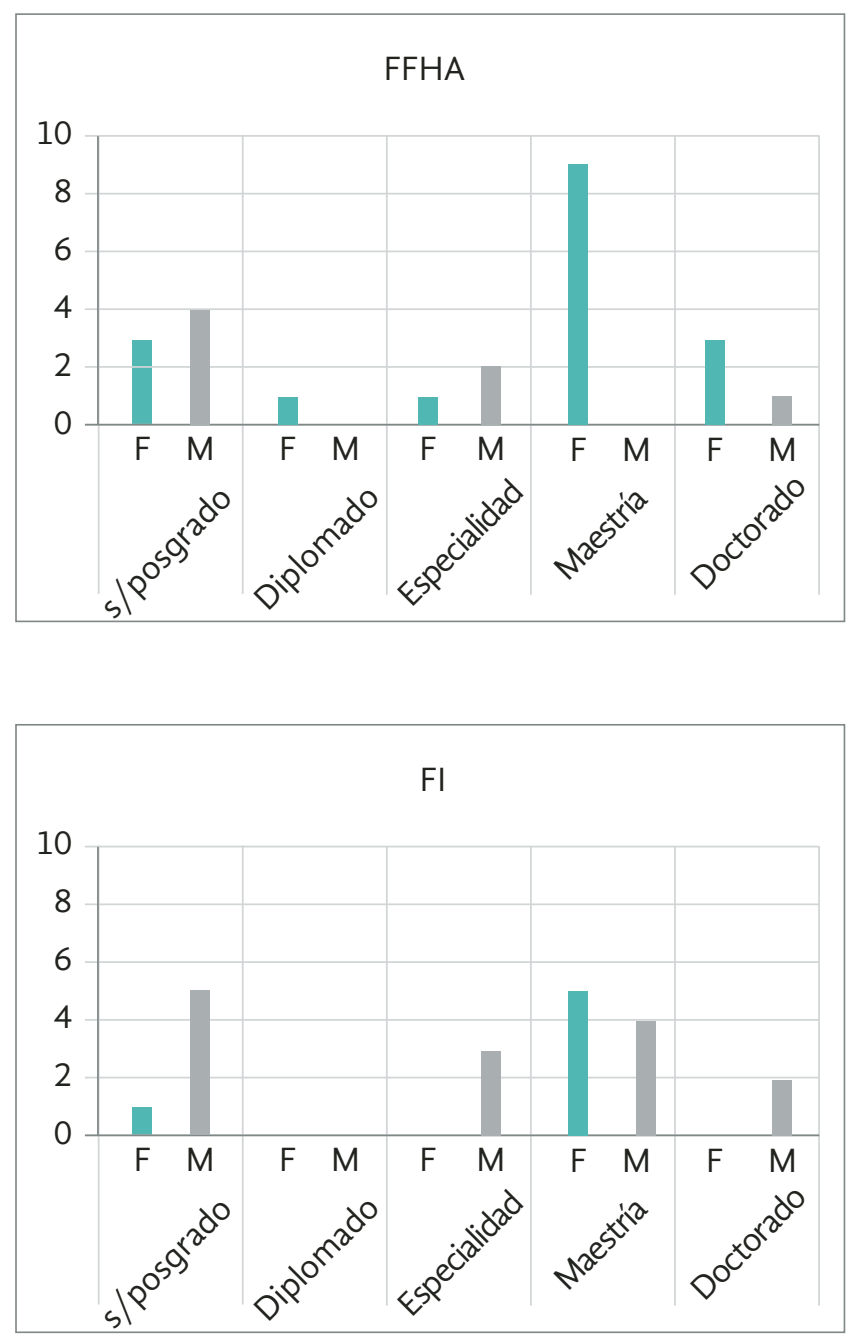

Fuente: Elaboración propia en base a la encuesta realizada a académicas/ os en gestión en la UNSJ.

para comprender las heterogeneidades presentes a la hora de conceptualizar el género. Las diversas miradas se presentan en lo cotidiano de manera compleja y relacional, donde los saberes propiamente científicos y del sentido común convergen para generar una definición que en primera instancia parece compartida por todos/as pero que cuando se profundiza, aparece difusa y conflictiva. 


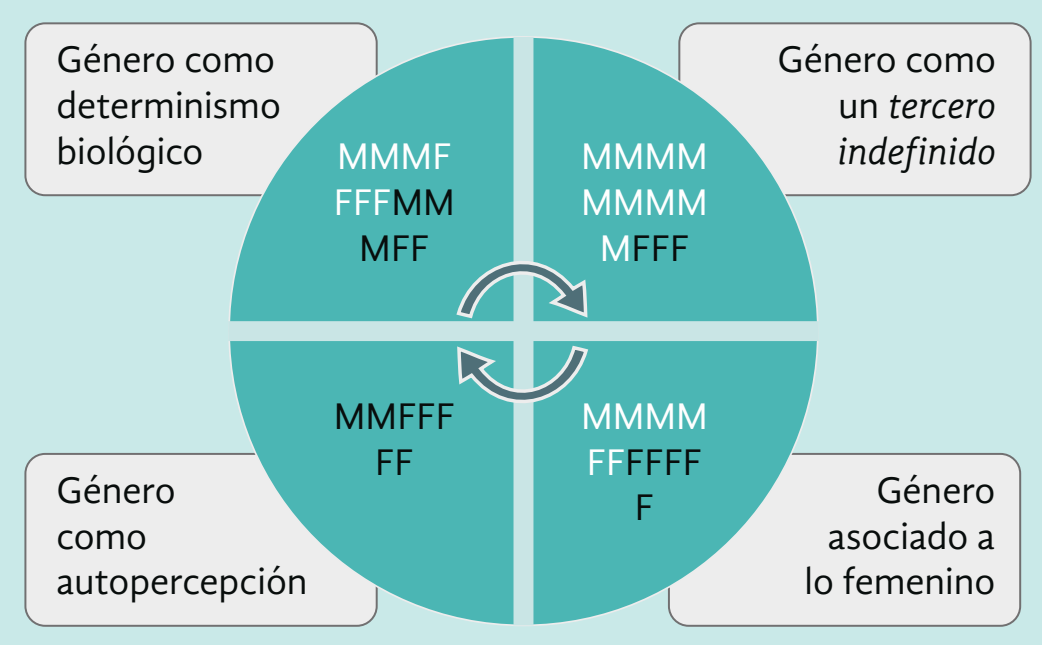

Referencias: $\mathrm{M}=$ Género masculino, $\mathrm{Fl} ; \mathrm{F}=$ Género femenino, Fl; $\mathrm{M}=$ Género masculino, FFHA; $\mathrm{F}=$ Género femenino, FFHA.

\section{Género como determinismo biológico}

El género como una construcción binaria es quizás una de las representaciones más convencionales de las sociedades contemporáneas. Aquí las expresiones de sentido de las personas que ocupan cargos de gestión denotan una equiparabilidad entre el binarismo de la sexualidad y la genitalidad (macho/hembra) con una identidad de género que es también entendida como binaria (masculino/femenino).

Las representaciones de los/las entrevistados/as que pudieron ser advertidas a través del estudio realizado no se desenvuelven en un vacío, son más bien el corolario de una simbolización cultural e histórica que se sostiene sobre la base de la diferencia anatómica de los cuerpos y que se expresa en un conjunto de prácticas y RS que otorgan una posición (objetiva y subjetiva) en relación al sexo.

El proceso de constitución del género conlleva una determinada forma de comprender lo femenino y lo masculino, lo que es distintivo de cada sexo. Esta idea subsume una de las discusiones contemporáneas acerca de lo que aglutina la mirada conservadora y patriarcal sobre el género; a saber; la idea de que los sexos son sólo dos (masculino/femenino), el supuesto que sostiene que las relaciones sexuales tienen como fin la procreación y, por último, que la familia es una unidad natural. Algunas de las respuestas de los entre- vistados/que nos permiten realizar estas apreciaciones enunciadas son las siguientes:

"El sexo de una persona. Si su forma de pensar, ser y sentir es acorde a su sexo. Creo... no lo tengo muy claro." (FI, F)

"De alguna manera me separa entre hombremujer (macho-hembra) desde un criterio básico donde las funciones son diferentes" (FI, M)

"Yo defino hombre y mujer; para mí no hay nada más. La representación general es hombre y mujer." (FF, M)

Se observa entonces que, como sostiene Lamas (1994) "En cada cultura la oposición binaria hombre/ mujer es clave en la trama de los procesos de significación. La diferencia sexual, recreada en el orden representacional, contribuye ideológicamente en la esencialización de la masculinidad y la feminidad; también produce efectos en el imaginario de las personas."

Sin embargo, entre quienes sostienen una mirada binaria evidenciamos una apertura a la idea de un concepto de género que podría llegar a ser no exclusivamente masculino y femenino.

"Género, femenino y masculino en principio, ya que ahora hay otras manifestaciones de género fuera de lo tradicional." (FI, F) 
"Lo entiendo como masculino o femenino/ varón-mujer. En estos tiempos mi mente se va abriendo y flexibilizando y entiendo que hay otras opciones." (FF, F)

Es plausible pensar que los debates actuales acerca de este tipo de temáticas y su consecuente difusión mediática hayan generado una representación que sanciona en términos sociales ciertas miradas rígidas que tienden a invisibilizar la diversidad como dimensión constitutiva del género.

Estas perspectivas que ponen en tensión la RS del género como binario muchas veces implican para quienes ocupan cargos de gestión adoptar una postura de sostenimiento de la igualdad de género como condición para la igualdad en la gestión.

"A veces a las mujeres se las deja de lado en algún tipo de tareas, pero ha cambiado bastante. Ahora creo que está más abierto para ellas." (FI, M)

"Tenemos mucha participación de mujeres y de varones para que haya armonía." (FI, M)

\section{Género como tercero indefinido}

En las expresiones de sentido aquí vertidas, encontramos que el tercero indefinido puede ser transgénero o no heterosexual, implicando de algún modo un debilitamiento de la RS conservadora y patriarcal que sostiene la mirada binaria.

El género entendido como indefinido aparece también aquí asociado a la idea de un "otro" que en términos históricos fue invisibilizado y que hoy como resultado del avance en materia de políticas públicas ${ }^{5}$ $\mathrm{y}$ derechos humanos ha generado que las personas que ocupan cargos de gestión deban aggiornar su discurso ante la existencia de un control/sanción social y legal que ampara múltiples identidades de género y orientaciones sexuales como sujetos de derechos.

"El género está dividido hoy en tres: hombre, mujer y un tercero." (FI, M)

"Es una forma de encarar la vida desde ser hombre o ser mujer o a través de una duda también.” (FF, F)

"Femenino, masculino, transgénero." (FI, M)
"Masculino, femenino, otro." (FF, F)

El avance en las políticas públicas y en la legislación vigente ha tenido su expresión institucional en la UNSJ. El Consejo Superior aprobó la creación en el año 2016 de la Oficina por la igualdad de género, contra las violencias y la discriminación y del Protocolo de actuación ante situaciones de discriminación, violencia a la identidad de género y contra las mujeres en el ámbito universitario.

Esta discusión a nivel institucional fue el caldo de cultivo para que en lo discursivo se sostenga una idea de igualdad y no discriminación, aunque sin dejar de reconocer que esta apertura puede significar un conflicto; un problema hacia adentro de la institución como espacio de trabajo, en otros términos, un debate no saldado entre lo que configura el ámbito de lo privado y de lo público.

"No conozco tanto, pero creo que tiene que ver con la igualdad, la no discriminación.” (FF, F)

"Las manifestaciones homosexuales en el trabajo no me molestan, aunque sé que en estos entornos las personas han tenido algunos conflictos." (FI, M)

El concepto de género es una construcción socio histórica que alude a un conjunto de prácticas, conductas, pautas y sentidos sobre lo que es definido por una sociedad dada como aceptable, permitido y obligatorio. En este sentido, todos aquellos sujetos que de alguna manera rompen con esas pautas son catalogados como poseedores de conductas desviadas o que infringen las normas sociales en su espacio y tiempo, lo que Howard Becker (2009) llamaría como outsiders.

"No hay que discriminar hay que respetarla y acompañarnos entre todos. Pero no hay que promocionar cosas que pueden degenerar o dañar a la sociedad, no hay que exagerar". (FI, M)

\section{Género como autopercepción}

La RS del género como autopercepción se apoya en la premisa de la variabilidad cultural e histórica del género, es decir, en la diferenciación del aspecto 
cultural y biológico en la que se basa la dicotomía sexo-género.

En las décadas del 70 y 80 la difusión del paradigma de la identidad de género trajo consigo un incremento de la investigación sobre las diferencias de sexo - género en los ámbitos académicos. Asimismo, las legislaciones en materia de identidad de género han permitido incorporar esta perspectiva a la hora de definir qué se entiende por género en las personas que ocupan cargos de gestión en la UNSJ.

En este sentido, el marco normativo ${ }^{6}$ en Argentina entiende por identidad de género a la vivencia interna e individual del género tal como cada persona la siente, la cual puede corresponder o no con el sexo asignado al momento del nacimiento, incluyendo la vivencia personal del cuerpo. Esto puede involucrar la modificación de la apariencia o la función corporal a través de medios farmacológicos, quirúrgicos o de otra índole, siempre que ello sea libremente escogido. También incluye otras expresiones de género, como la vestimenta, el modo de hablar y los modales.

De esta definición contenida en la ley se desprende una idea de identidad de género asociada a la autopercepción del ser humano y que se expresa en el lenguaje de los actores objeto de esta investigación.

“...una persona tiene la posibilidad de elegir cómo sentirse mejor representado. Va mucho más allá de lo sexual.” (FF, F)

"Es una construcción, no tiene que ver con el sexo biológico. Es una construcción cultural que está ajustada a la época y al contexto.” (FF, F)

"Supera la idea de sexo, es más amplio y da la posibilidad a las personas de buscar su identificación." (FF, M)

\section{Género asociado a lo femenino}

Aquí subyacen RS que vinculan el género como lo femenino de manera exclusiva. En términos históricos el movimiento feminista en los años 70 comienza a poner en el eje del debate el género como una construcción socio-cultural y política, para dar batalla a los estereotipos vinculados a la identidad masculina como hegemónica y a la femenina como subordinada. 


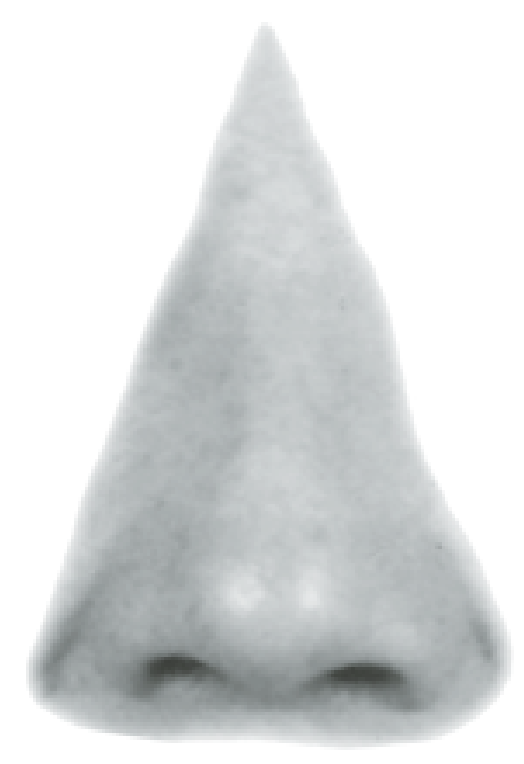

Con el devenir de la historia, esos cuerpos de ideas disruptivas terminan siendo consideradas como sinónimo de un acontecer meramente femenino. Esto no se presenta de manera aleatoria, más bien todo lo contrario, las disputas en el campo del género son siempre y antes que nada representaciones del poder ${ }^{5}$, de la relación de lo público y lo privado y los roles adjudicados a cada género.

"Género femenino, tal vez está referido a varones y mujeres, pero una siempre lo tomó como referido a la mujer." (FI, F)

"El género femenino es lo mejor (...) Abarca la pluralidad que representan las mujeres.” (FF, F)

"(...) el género lo veo como el mundo femenino." (FI, M)

Por su parte, el género como autopercepción se apoya en la premisa de la variabilidad cultural e histórica del género, es decir, en la diferenciación del aspecto cultural y biológico en la que se basa la dicotomía sexo-género.

"El mundo de lo femenino" construido mediáticamente a través de revistas y redes sociales que lo explotan y generan un conjunto de miradas y estereotipos que condensan la idea de género asociado a las mujeres, como el único destino de lo posible. Y, junto a ello, una representación colectiva que subsume un conjunto de rasgos y disposiciones socialmente aceptadas de lo que implica ser mujeres contemporáneas.

En este sentido, quienes ocupan cargos de gestión en la UNSJ se desarrollan su vida en el ámbito de lo estrictamente académico, pero, en tanto sujetos sociales no están exentos de las perspectivas que propician determinadas RS sobre el género.

El conocimiento es un sistema de saberes situados y las RS que poseen quienes ocupan cargos de gestión en la UNSJ está permeada por el contexto histórico social en la que se desenvuelven determinadas problemáticas sociales. 
"Género relacionado con la violencia de género, estamos todos involucrados.” (FI, F)

"La violencia de género está en todos los contextos sociales." (FF, F)

"En la actualidad las mujeres luchan por igualdad de derechos, donde los varones son género dominante." (FI, M)

"Está muy candente la pelea por los derechos de las mujeres, por el cese de la violencia de género." (FF, F)

En Argentina, el debate acerca de las problemáticas de las mujeres tiene una historia nutrida. La realización de manera continuada del Encuentro Nacional de Mujeres desde 1984, el Paro Internacional de Mujeres cada 8 de marzo en el marco de las reivindicaciones por el día Internacional de la Mujer, la organización de la lucha contra la violencia de género que abraza el colectivo "Ni Una Menos" desde el 3 de junio de 2015 y el pedido de cese de la violencia machista y de la complicidad estatal, reclamos de prevención, cuidado, igualdad y justicia son el escenario en el que discurren muchas de las RS asociadas al género como femenino, vinculado directamente a la violencia de género y a la lucha feminista.

\section{Reflexiones finales}

El propósito de las reflexiones aquí vertidas apuntó a reconstruir las significaciones y sentidos que las personas que ejercen la gestión académica en el período 2016-2020 en la FI y la FFHA pertenecientes a la UNSJ, tienen sobre el concepto de género.

Dicho propósito se vio orientado por un supuesto de investigación que sostiene que las RS sobre el concepto de género de las personas que ocupan cargos de gestión varía en función de la formación disciplinar obtenida.

En base a las aportaciones del enfoque de las RS construimos un sistema de categorías para comprender las similitudes y diferencias presentes a la hora de conceptualizar el género al interior de la UNSJ. Las diversas miradas se presentan en lo cotidiano de manera compleja y relacional, donde los saberes propiamente científicos y del sentido común convergen para generar una definición que en primera instancia parece compartida por todos/as pero que cuando se profundiza, aparece difusa y conflictiva.

En este sentido, observamos que en torno al concepto de género discurren diversas RS. El género como un determinismo biológico subsume una de las discusiones contemporáneas acerca de lo que aglutina la mirada conservadora y patriarcal sobre el género; a saber; la idea de que los sexos son sólo dos (masculino/ femenino). Las expresiones de sentido dan cuenta de una paridad de personas en gestión de ambas facultades de la UNSJ que consideran al género de esta manera.

Por otro lado, el género como tercero indefinido aparece asociado a la representación de un "otro" invisibilizado históricamente. Aquí encontramos expresiones de sentido que se refieren tanto a personas transgénero o no heterosexuales. El avance en materia de políticas públicas y derechos humanos generó que las personas que ocupan cargos de gestión deban aggiornar su discurso ante la existencia de un control/sanción social y legal que ampara múltiples identidades de género y orientaciones sexuales como sujetos de derechos. Los relatos que van en este sentido corresponden mayoritariamente a varones de la FI.

Por su parte, el género como autopercepción se apoya en la premisa de la variabilidad cultural e histórica del género, es decir, en la diferenciación del aspecto cultural y biológico en la que se basa la dicotomía sexo-género. Esta mirada se sostiene normativamente en la sanción de la Ley de Identidad de Género en la Argentina, permeando los discursos de las personas que ocupan cargos de gestión en la UNSJ. Quienes adhieren a esta mirada sobre el género pertenecen exclusivamente a la FFHA.

Por último, el género asociado a lo femenino aglutina un conjunto de estereotipos que condensan este concepto asociado exclusivamente a las mujeres, como el único destino de lo posible. Y, junto a ello, una representación colectiva que subsume un conjunto de rasgos y disposiciones socialmente aceptadas de lo que implica ser mujeres contemporáneas.

A su vez esta última representación se encuentra permeada por la emergencia mediática y social de la problemática de género en la sociedad argentina, en otros términos, las disputas en el campo del género son siempre y antes que nada representaciones del poder, de la relación de lo público y lo privado y los 
roles adjudicados de manera diferencial. Las expresiones de sentido dan cuenta de una paridad de personas en cargos de gestión de ambas facultades de la UNSJ que consideran al género asociado exclusivamente a lo femenino.

De esta manera, la idea que sostiene que las RS sobre el concepto de género de las personas que ocupan cargos de gestión en la UNSJ varía en función de la formación disciplinar obtenida es puesto en cuestión por el presente trabajo.

Más allá de los cargos de gestión que ocupan y la pertenencia institucional a una facultad específica su RS acerca del género va más allá de la universidad como agente formadora de sujetos sociales. Las personas aquí entrevistadas se desenvuelven en otros espacios que trascienden a la universidad y que dan forma a su manera de conceptualizar el género.

Las reflexiones aquí vertidas no son lineales, ello implica no desconocer que los enfoques hegemónicos en torno al género son el resultado de una disputa de poder que instala determinados discursos por sobre otros. En este sentido, la formación académica aparece tamizando estas miradas, poniéndolas en cuestión a través de espacios de discusión y debate que muchas veces exceden lo estrictamente académico.

Por otro lado, es importante destacar que en repetidas ocasiones la formación académica aparece a nivel de lo discursivo como un catalizador en el acceso a cargos de gestión académica. En el caso de la FI y FFHA el género emergió como un determinante de mayor peso ya que si bien existe una mayor cantidad de mujeres con formación de posgrado estas acceden a cargos de menor jerarquía.

En suma, hay una diferenciación marcada en lo referente al universo de sentidos y al universo de las prácticas para quienes ocupan cargos de gestión en la FI y en la FFHA de la UNSJ, una disputa no resuelta entre el ámbito de lo público, en tanto cargos electivos, y en el ámbito de lo privado, en tanto sujetos sociales permeados por los debates actuales en torno al género.
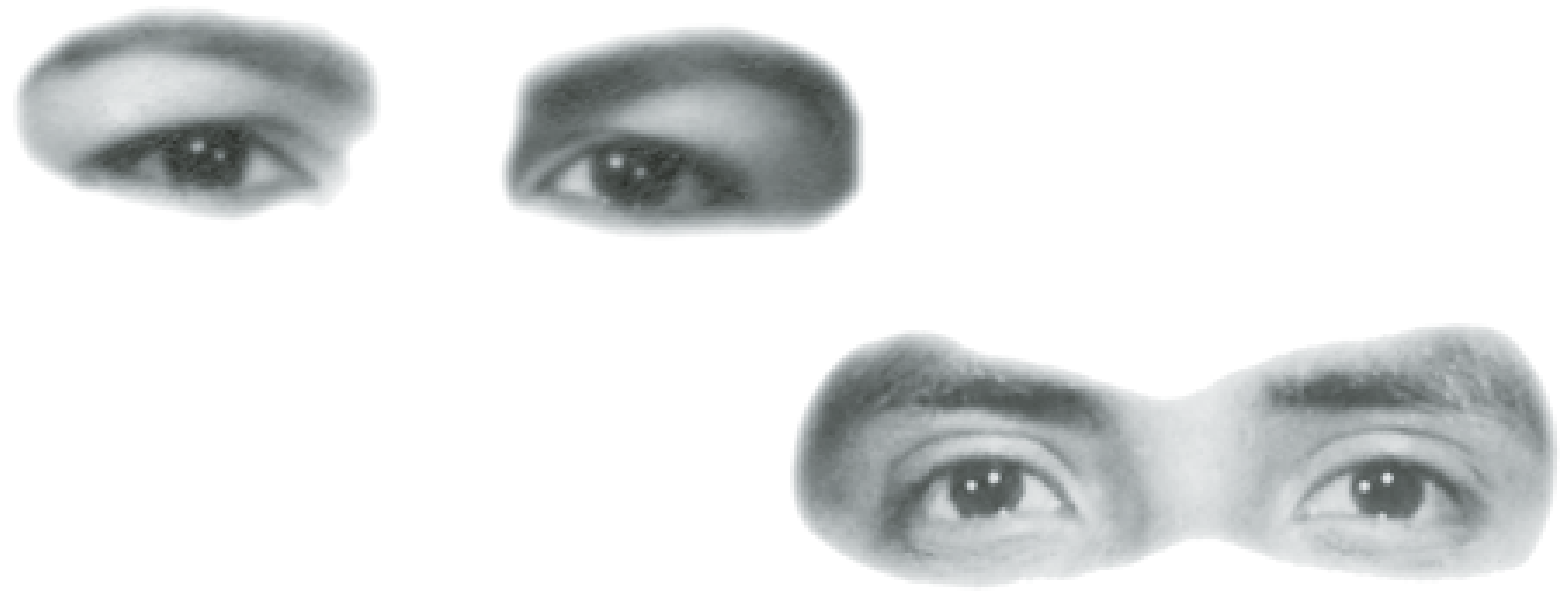


\section{Notas}

1. Cuando en el presente trabajo se refiera a personas en gestión académica es pertinente aclarar que, a los fines del trabajo de investigación, sólo se considera a los cargos electivos, excluyendo las secretarías de cada facultad, que, si bien son personas en gestión académica, no son cargos designados por medio de elecciones.

2. En torno al concepto de Género se advierte que hay diversos tratamientos sobre este e incluso una fuerte crítica por parte del Feminismo Decolonial. María Lugones sostiene que éste no existía en AbyaYala y que es una invención del Colonialismo. Así, la propuesta de Lugones en cuanto decolonial plantea la (in)existencia de género, es decir pone en tensión y perturba las categorías dualistas jerárquicas, heteronormativas y heterosexualistas de: hombre y mujer, macho y hembra (Lugones, 2008, 2012). Más allá de esta profunda crítica al Sistema Moderno/Colonial de Género, con la cual se acuerda, aún no se encuentra otro concepto superador del mismo.

3. Expresión de ello es la creación en el año 2016 de la Oficina por la igualdad de género, contra las violencias y la discriminación y del Protocolo de actuación ante situaciones de discriminación, violencia a la identidad de Género y contra las mujeres en el ámbito universitario; así como también las discusiones que están teniendo lugar en el marco de la Conmemoración de los 100 años de la Reforma Universitaria de 1918.

\section{Bibliografía}

Anzorena, C. (2008). "Estado y división sexual del trabajo: las relaciones de género en las nuevas condiciones del mercado laboral", en Utopía y Praxis Latinoamericana, año 13, núm. 41. (Abril-Junio, 2008). CESA - FACES - Universidad del Zulia, Maracaibo.

Becker, H. (2009). Outsiders. Hacia una sociología de la desviación, Siglo XXI Editores, Buenos Aires.

Blázquez Graf, N.; Flores Palacios, F. y Ríos Everardo, M. (2012). Investigación Feminista:Epistemología, Metodología y Representaciones Sociales, Centro de Investigaciones Interdisciplinares en Ciencias y Humanidades. Centro Regional de Investigaciones Multidisciplinarias, Universidad Nacional Autónoma de México, México.

Celiberti, L. y Mesa, S. (2009). Las relaciones de género en el trabajo productivo y reproductivo, IPS, Montevideo, Uruguay.

Durkheim, E. (2010). Las reglas del método sociológico, Ediciones Libertador, Buenos Aires, Argentina.

Faur, E. (2004). Masculinidades y desarrollo social. Las relaciones de género desde la perspectiva de los hombres, UNICEF, Arango Editores Ltda., Colombia, Bogotá.

Heller, L. (2011). Mujeres en la Cumbre Corporativa: El Caso de la Argentina. Revista del Centro de Estudios de Sociología del Trabajo. núm. 3. abril de 2011, Buenos Aires, Argentina. Facultad de Ciencias Económicas. Universidad de Buenos Aires.

Hewlett, S. (2006). Off Ramps and On Ramps: Keeping Talented Women on the Road to Success. Harvard Business School Press.

Jodelet, D. (1988). “Las Representaciones Sociales” en S. Moscovici. Psicología Social, II. Pensamiento y vida social. Psicología social y problemas sociales, Paidós, Bs. As., Argentina.

Lamas, M. (1994). Cuerpo, diferencia sexual y género en Revista Debate Feminista. $\mathrm{N}^{\circ} 10$, México.
4. Cabe señalar que no se discrimina por edad, formación, antigüedad en el cargo y cargo que actualmente ocupa, atendiendo al criterio de confidencialidad y anonimato de los/las entrevistados/as considerando que la universidad en estudio es una institución con un número reducido de cargos de gestión, por lo que sería fácilmente individualizada la unidad de análisis.

5. Ley 26.485: Ley de Protección Integral a las Mujeres.

http://servicios.infoleg.gob.ar/infolegInternet/anexos/150000-154999/152155/norma.

Ley 26.743: Identidad de Género.

http://servicios.infoleg.gob.ar/infolegInternet/anexos/195000-199999/197860/norma.htm

Ley No 23.592 - Actos Discriminatorios.

http://servicios.infoleg.gob.ar/infolegInternet/anexos/20000-24999/20465/texact.htm

6. Artículo $2^{\circ}$ de la Ley 26.743: Identidad de Género. Sancionada el 9 de mayo de 2012.

7. Carol Hanisch (1969) "Lo personal es político" en el marco de un sistema de dominación patriarcal y capitalista.

Lugones, M. (2008). "Colonialidad y género: hacia un feminismo descolonial” en Mignolo, W., Lugones, M. Lucena, I y Tlostanova, M. V. Género y descolonialidad, Ediciones del Signo, Bs. As., Argentina.

(2012). Subjetividad esclava, colonialidad de género, marginalidad y opresiones múltiples. Pensando los feminismos en Bolivia, disponible en http://rcci.net/globalizacion/2013/fg1576.htm

Martínez Ruiz, M. A. Merma Molina G. Gavilán Martín D., (2013). "Redes colaborativas del profesorado: Proyecto diseño y atención a las oportunidades de género en la educación superior". RIIEP. Vol. 6, núm. 2 julio-diciembre de 2013, pp. 161-172.

Moine, M. B. (2013). Factores que inciden en el acceso de las mujeres a cargos de responsabilidad en la estructura organizacional. Ciencias económicas 10.01 / páginas 21-45 / Investigación.

Pautassi, L.C. (2000). "Igualdad de derechos y desigualdad de oportunidades: Ciudadanía, derechos sociales y género en América Latina" en Gioconda Herrera (coord.). Las fisuras del patriarcado. Reflexiones sobre feminismo y derecho, Flacso, Ecuador.

(2011). "La igualdad en espera: el enfoque de género" en Lecciones y Ensayos, núm. 89, 2011, pp. 279-298.

Pérez Rubio, A. M. y Saavedra, G. (2002). "De los discursos y las prácticas: un ejemplo de aplicación del modelo de las representaciones sociales" en Comunicación y Sociedad, núm. 39, Universidad de Guadalajara. México, pp. 179-204.

Universidad Nacional de San Juan (2016): Ordenanza N. ${ }^{0}$ 019/2016-CS, de creación de la "Oficina por la igualdad de género, contra las violencias y la discriminación" y de aprobación del "Protocolo de actuación ante situaciones de discriminación, violencias a la identidad de género y contra las mujeres en la UNSJ". Disponible en: http://www.digesto. unsj.edu.ar/admin/digesto_admin/pdf/ORD19O-1611921.pdf

Vasilachis de Gialdino, I. (1992): Métodos Cualitativos: Los problemas teórico-epistemológicos, Centro Editor de América Latina, Buenos Aires. 